\title{
MS18-P15 | Thermochromic Lead-Free Halide Double Perovskites
}

Ning, Weihua (Linkoping university, Linköping, SWE); Gao, Feng (Linkoping university, Linkoping, SWE)

Lead-free halide double perovskites with diverse electronic structures andoptical responses, as well as superior material stability show great promise for a range of optoelectronic applications. However, their large bandgaps limit their applications in the visible light range such as solar cells. In this work, an efficient temperature-derived bandgap modulation, that is, an exotic fully reversible thermochromism in both single crystals and thin films of Cs2Ag-BiBr6 double perovskites is demonstrated. Along with the thermochromism, temperature-dependent changes in the bond lengths of $\mathrm{Ag}-\mathrm{Br}\left(\mathrm{R}_{\mathrm{Ag}-\mathrm{Br}}\right)$ and $\mathrm{Bi}-\mathrm{Br}_{(\mathrm{RBi}-\mathrm{Br})}$ are observed. The first-principle molecular dynamics simulations reveal substantial anharmonic fluctuations of the $\mathrm{R}_{\mathrm{Ag}-\mathrm{Br}}$ and $\mathrm{R}_{\mathrm{Bi}-\mathrm{Br}}$ at high temperatures. The synergy of anharmonic fluctuations and associated electron-phonon coupling, and the peculiar spin-orbit coupling effect, is responsible for the thermochromism. In addition, the intrinsic bandgap of $\mathrm{Cs}_{2} \mathrm{AgBiBr}_{6}$ shows negligible changes after repeated heating/cooling cycles under ambient conditions, indicating excellent thermal and environmental stability. This work demonstrates a stable thermochromic lead-free double perovskite that has great potential in the applications of smart windows and temperature sensors. Moreover, the findings on the structure modulationinduced bandgap narrowing of $\mathrm{Cs}_{2} \mathrm{AgBiBr}_{6}$ provide new insights for the further development of optoelectronic devices based on the lead-free halide double perovskites. 